\title{
EVALUATION OF THE FRONT-FIXING METHOD CAPABILITIES FOR NUMERICAL MODELLING OF DIFFUSION IN MOVING SYSTEMS
}

\author{
I. O. Golosnoy, J. K. Sykulski \\ Electrical Power Engineering Research Group, School of Electronics and Computer Science, \\ University of Southampton, Highfield, Southampton, SO17 1BJ, UK \\ ig@ecs.soton.ac.uk, jks@soton.ac.uk
}

Keywords: diffusion; numerical modelling; movement

\begin{abstract}
Application of a finite difference front fixing method to various problems with moving boundaries and nonlinear material properties is discussed. Advantages and implementation problems of the method are highlighted. Particular attention is given to conservation properties of the algorithm and accurate solutions close to the moving boundaries. The algorithm is tested using a well-known solution of the plane diffusion problem with complex conditions at the moving interface.
\end{abstract}

\section{Introduction}

Diffusion equations are widely used to describe electromagnetic processes, e.g. electrical machines motion or eddy currents. Formulations of field variations in terms of diffusion of the magnetic vector potential allow to utilise many numerical methods developed previously for heat and mass transport [5]. Often the modelling of the diffusion equation encounters problems with strong non-linearity due to extreme variations in material properties with field changes, e.g. high-temperature super-conductivity [4], or with magnetic phase transitions due to Joule heating [3]. For such dynamic problems with strong non-linearity the finite differences methods usually deliver adequate description of the localised phenomena. Moreover, developments of finite volume techniques, together with $\mathrm{ADI}$ and fractional step methods [5], provide powerful tools for constructing unconditionally stable, high order approximations at irregular boundary nodes even for complex 3D systems.

The paper deals with modelling of diffusion in an abstract system with a moving boundary. The motion is assumed to be a function of the solution itself and many numerical methods developed previously [1] are not able to cope with such a strong coupling. On the other hand, the transformations proposed by Landau [1] do introduce a co-ordinate system in which all of the spatial boundaries are fixed. Under the transformation the new computational domains remain the same with an additional non-linear equation for the boundary motion. This allows keeping nodes close to the interface independent of the motion. Of course, there is a price for such an improvement: the conservation laws in the new system must be modelled carefully and iterations are required to solve non-linear coupling between field diffusion and the interface displacement. This may be assessed using a simplified plane system. The main challenges are the implementation of conservation laws at the moving boundaries and an effective mesh refinement. These issues are addressed in the paper.

\section{Front-fixing method for diffusion problems}

\subsection{Plane diffusional system}

A diffusion-controlled phase change is an example of a non-linear, coupled diffusion-motion problem with a discontinuity at the moving interface. It can be described by variation of an abstract potential or field strength $(\phi)$ with position $(x)$. Potential profiles depend on time $(t)$. Diffusive processes occur simultaneously in two distinct domains $(A \& B)$. But the potential of one domain in contact with the other is fixed by a thermodynamic constraint (equilibrium potentials $\phi_{A}$ and $\phi_{B}$ ). The rates at which potential diffuses towards the interface through $A$ and is removed into $B$ are not necessarily equal. In order to conserve energy the interface between the two domains must move. Writing the interface position as $s=s(t)$, the following set of coupled non-linear differential equations may be used to model the system [1]

$$
\begin{gathered}
\frac{\partial \phi}{\partial t}=\frac{\partial}{\partial x}\left(D(\phi(x, t)) \frac{\partial \phi(x, t)}{\partial x}\right), 0<x<L, x \neq s(t) \\
\left.D_{A} \frac{\partial \phi}{\partial x}\right|_{s(t)^{-}}-\left.D_{B} \frac{\partial \phi}{\partial x}\right|_{s(t)^{+}}=\left(\phi_{B}-\phi_{A}\right) \frac{d s(t)}{d t}, x=s(t)
\end{gathered}
$$

\subsection{Front fixing, interface and implementation}

Landau transformation utilises new positional variables (one for each domain). Introduction of $u=x / s(t)$ fixes the extent of $A$ to the domain $0 \leq u \leq 1$, while $v=(x-s) /(L-s)$ fixes the extent of $B$ to the domain $0 \leq v \leq 1$. The divergent form of the diffusion Equation (1) and interface Equation (2) may be written as [2]:

$$
\begin{gathered}
\frac{\partial(s \phi)}{\partial t}=\dot{s} \frac{\partial(u \phi)}{\partial u}+\frac{1}{s} \frac{\partial}{\partial u}\left(D_{A} \frac{\partial \phi(u, t)}{\partial u}\right), \dot{s} \equiv \frac{d s}{d t}, 0<u<1 \\
\frac{\partial((L-s) \phi)}{\partial t}=\dot{s} \frac{\partial((1-v) \phi)}{\partial v}+\frac{1}{L-s} \frac{\partial}{\partial v}\left(D_{B} \frac{\partial \phi(v, t)}{\partial v}\right), 0<v<1 \\
\left.\frac{D_{A}}{s} \frac{\partial \phi}{\partial u}\right|_{u=1}-\left.\frac{D_{B}}{L-s} \frac{\partial \phi}{\partial v}\right|_{v=0}=\left(\phi_{B}-\phi_{A}\right) \frac{d s(t)}{d t}
\end{gathered}
$$


In order to derive a finite difference scheme, space is discretised at $M+1$ points. The first $N+1$ points are given by a fixed discretisation of $u$, which corresponds to the extent of $A$. Using a subscript notation to denote discretisations of space, the points in $A$ are written as $u_{0}=0, u_{1}, \ldots, u_{N}=1$. The last $M-N+1$ points are in $B$ as given by a fixed discretisation $v_{N}=0, v_{N+1}, \ldots, v_{M}=1$. Discretisations of time are indicated using superscripts (e.g. $t^{j}$ ). Integrations of Equation (3) around each node $i$ over one time step provide a set of conservative equations in the bulk material, e.g. for $A$ :

$$
\begin{aligned}
& \left(\phi_{i}^{j+1} s^{j+1}-\phi_{i}^{j} s^{j}\right)\left(u_{i+0.5}-u_{i-0.5}\right)= \\
& =\left(s^{j+1}-s^{j}\right)\left(\phi_{i+0.5}^{j+1} u_{i+0.5}-\phi_{i-0.5}^{j+1} u_{i-0.5}\right)+ \\
& +\frac{t^{j+1}-t^{j}}{s^{j+1}}\left(D_{A} \frac{\phi_{i+1}^{j+1}-\phi_{i}^{j+1}}{u_{i+1}-u_{i}}-D_{A} \frac{\phi_{i}^{j+1}-\phi_{i-1}^{j+1}}{u_{i}-u_{i-1}}\right)
\end{aligned}
$$

The interface Equation (4) is approximated in a consistent way to conserve energy [2] $\left(\delta t=t^{j+1}-t^{j}\right)$ :

$$
\begin{aligned}
& \frac{D_{B} \delta t}{L-s^{j+1}}\left(\frac{\phi_{N+1}^{j+1}-\phi_{B}}{v_{N+1}}\right)-\frac{D_{A} \delta t}{s^{j+1}}\left(\frac{\phi_{A}-\phi_{N-1}^{j+1}}{1-u_{N-1}}\right)=\left(s^{j+1}-s^{j}\right) \times \\
& \left(\phi_{N-0.5}^{j+1} \frac{1+u_{N-1}}{2}+\phi_{A} \frac{1-u_{N-1}}{2}-\phi_{N+0.5}^{j+1}\left(1-\frac{v_{N+1}}{2}\right)-\phi_{B} \frac{v_{N+1}}{2}\right)
\end{aligned}
$$

The set of simultaneous equations involves $M+3$ unknowns, i.e. the future potentials and the future interface position $s^{j+1}$ (with 4 values fixed by the boundary conditions). Since all of the equations are coupled, if the implicit scheme is to conserve energy, the entire system must be solved simultaneously. But the fact that they form a non-linear system means that this is potentially very demanding in terms of computing times. It is interesting to note, however, that Equations (5) and (6) are only weakly coupled; thus, if the future interface position $s^{j+1}$ were known, the diffusion problem (5) would become linear. Conversely, if future potentials were known, the future interface position could be calculated from Equation (6) relatively easily. It is possible to implement an efficient algorithm based on de-coupling the problem in this way. Normally it requires only 3 to 4 iterations to reach a consistent solution.

\subsection{Validation}

It is confirmed that the numerical scheme conserves energy (within rounding accuracy) in every calculation. The question of whether the solution is accurate remains. For particular initial and boundary conditions, analytical solutions are available [1]. In this case the interface position should vary with the square root of time. This behaviour is reflected in Figure 1 (for geometry where $L=1, D=1$ ). One advantage of discretising the problem in the transformed space is that the meshes automatically adjust themselves to accommodate the moving interface position. It is therefore possible to impose irregular meshes with fine resolution in regions where large concentration gradients are expected (near to the interface, for example) and larger space steps elsewhere. The results plotted in Figure 1 correspond to calculations completed using both irregular and regular meshes. It is clear that, for a given number of discretisation points, it is possible to find significantly more accurate solutions by using irregular meshes. In this way errors can be reduced without requiring any extra computational effort.

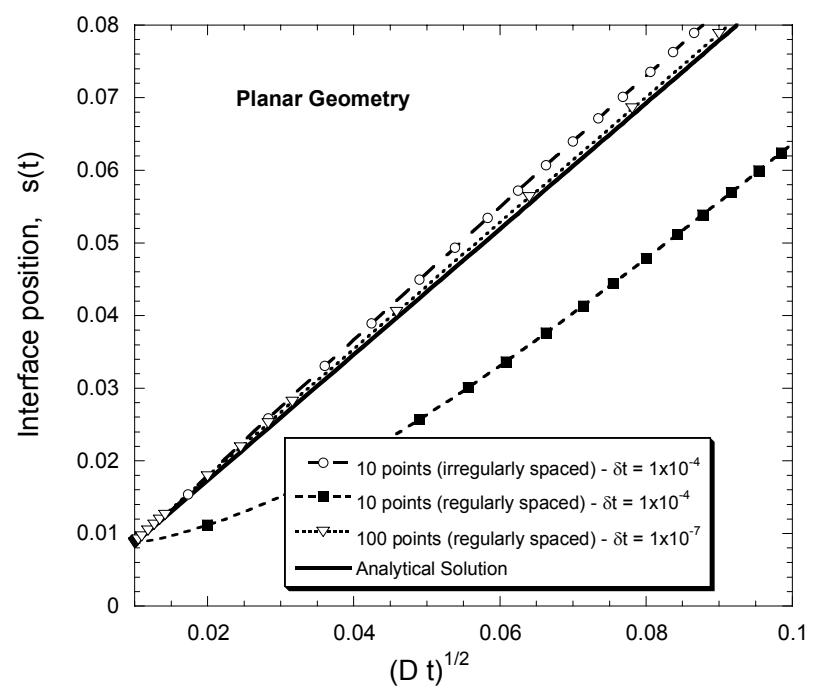

Figure 1. Analytical and numerical predictions for interface position: mesh size and time step effects [2].

\section{Conclusions}

It is suggested to use a front-fixing method for modelling of non-linear diffusion processes in various electromagnetic phenomena. Potential problems with implementation of conservation laws and complex boundary conditions in the front-fixing method are considered and solutions are suggested. It is shown that high accuracy can be achieved on a coarse irregular mesh since the interface is fixed in new coordinates.

\section{References}

[1] J. Crank. "Free and Moving Boundary Problems". Clarendon Press, (1984).

[2] T. C. Illingworth and I. O. Golosnoy. "Numerical Solutions of Diffusion-Controlled Moving Boundary Problems which Conserve Solute", Journal of Comp. Physics, 209(1), pp. 207-225, (2005).

[3] Y. B. Kudasov. "Magnetic field diffusion during a metal-insulator phase transition ", Technical Physics 43(12), pp. 1440-1444, (1998).

[4] J. K. Sykulski, M. Rotaru, R. L. Stoll. "2D Modeling of Field Diffusion and $A C$ Losses in High Temperature Superconducting Tapes", IEEE Trans. on Magnetics, 36(4), pp. 1178-1182, (2000).

[5] J. C. Tannehill, D. A. Anderson, R. H. Pletcher. "Computational Fluid Mechanics and Heat Transfer". 2nd ed., Taylor \& Francis, 792p. (1997). 